\begin{tabular}{|c|l|}
\hline Title & Pair of excitable FitzHugh-Nagumo elements: Synchronization, multistability, and chaos \\
\hline Author(s) & Y anagita, T.; Ichinomiya, T.; Oyama, Y. \\
\hline Citation & $\begin{array}{l}\text { PHY SICAL REVIEW E, 72,056218 } \\
\text { https://doi.org/L0.1103/PhysRevE.72.056218 }\end{array}$ \\
\hline Issue Date & 2005 \\
\hline Doc URL & http://hdl.handle.net/2115/5749 \\
\hline Rights & Copyright $\odot 2005$ A merican Physical Society \\
\hline Type & article \\
\hline File Information & PRE72-5.pdf \\
\hline
\end{tabular}

Instructions for use 


\title{
Pair of excitable FitzHugh-Nagumo elements: Synchronization, multistability, and chaos
}

\author{
T. Yanagita, ${ }^{*}$ T. Ichinomiya, ${ }^{\dagger}$ and Y. Oyama ${ }^{\ddagger}$ \\ Laboratory of Nonlinear Studies and Computation, Research Institute for Electronic Science, Hokkaido University, \\ Sapporo 060-0812, Japan
}

(Received 27 January 2005; revised manuscript received 25 May 2005; published 28 November 2005)

\begin{abstract}
We analyze a pair of excitable FitzHugh-Nagumo elements, each of which is coupled repulsively. While the rest state for each element is globally stable for a phase-attractive coupling, various firing patterns, including cyclic and chaotic firing patterns, exist in an phase-repulsive coupling region. Although the rest state becomes linearly unstable via a Hopf bifurcation, periodic solutions associated to the firing patterns is not connected to the Hopf bifurcation. This means that the solution branch emanating from the Hopf bifurcation is subcritical and unstable for any coupling strength. Various types of cyclic firing patterns emerge suddenly through saddle-node bifurcations. The parameter region in which different periodic solutions coexist is also found.
\end{abstract}

DOI: 10.1103/PhysRevE.72.056218

\section{INTRODUCTION}

Numerous chemical [1,2] and biological [3] phenomena can be modeled in the framework of reaction-diffusion systems, which show an unexpectedly rich variety of dynamical behaviors. In particular, excitability is a key property of many physical systems and plays a fundamental role in neural information processing and many other biological systems.

A single neuron displays excitable behavior, in the sense that a small perturbation to its quiescent state, i.e., a stable stationary value of the cross membrane potential, can lead to a large excursion of its potential. Such generation of a single spike in the electrical potential across the neuron membrane is a typical example of the excitable behavior. Excitable units usually appear as constitutive elements of complex systems, and can transmit excitation between them. The dynamics of the system depends crucially on the properties of each unit and on their interactions.

Periodic sequences of neural impulses, such as rhythmic firing patterns produced by central pattern generators, are of fundamental significance for the control of dynamic functions of the body. It is great interest to understand the mechanism of neural networks which cause and sustain such a wide variety of periodic activities [4]. These complex firing patterns including chaotic firings are modeled through the effect of high dimensional dynamics of an individual element, interaction of many neurons with spatial degree of freedom, and time delayed coupling [5-8]. There are many studies for excitable elements under external periodic stimuli, both experimentally and theoretically [9-13], which show various behaviors including phase-locking and chaotic behaviors. It has been also studied for an ensemble of oscillatory or excitatory elements interacting each other in the context of synchronization and chaos $[2,5-7,14]$. Here, we focus on a "minimal" model that consists of repulsively coupled two excitable neurons. We report that this model produces and

\footnotetext{
*Electronic address: yanagita@nsc.es.hokudai.ac.jp

${ }^{\dagger}$ Electronic address: miya@nsc.es.hokudai.ac.jp

"Electronic address: oyama@nsc.es.hokudai.ac.jp
}

PACS number(s): 05.45.Xt, 82.40.Bj

sustains various firing patterns: there are many types of sequence of firing while only two neurons are involved in the system.

For a system of excitable elements with diffusive coupling that activates each other, the rest state is globally stable: a finite perturbation to the rest state causes the excitation of an element. The excitation of the element is transmitted to the other element, then, the excitation of the other element follows immediately. And the phase difference between these excitations decreases due to the phaseattractive interaction, which comes from the diffusive coupling with positive coefficient between neurons. Then the synchronization occurs and the elements return to the rest state. Therefore we need external inputs so that dynamical behaviors appear in excitable elements with phase-attractive interaction.

The diffusive coupling with a negative coefficient has been considered as the effect of phase-repulsive coupling on two dimensional coupled FHN arrays [15]. The periodic firings are found in two neurons with excitatory and inhibitory synaptic couplings [16-18]. In this paper, we consider a rather mathematical model, a pair of excitable neurons with phase-repulsively coupling. We find that a pair of simple excitable elements shows various firing patterns including chaotic firing when elements interact phase-repulsively.

\section{MODEL}

In the following, we study a pair of excitable neurons, which are modeled by the FitzHugh-Nagumo (FHN) excitable elements $[19,20]$, given by the following equations:

$$
\begin{gathered}
\frac{d u_{1}}{d t}=u_{1}\left(u_{1}-\alpha\right)\left(1-u_{1}\right)-v_{1}+\frac{K}{2}\left(u_{2}-u_{1}\right), \\
\frac{d v_{1}}{d t}=\tau\left(u_{1}-\gamma v_{1}\right), \\
\frac{d u_{2}}{d t}=u_{2}\left(u_{2}-\alpha\right)\left(1-u_{2}\right)-v_{2}+\frac{K}{2}\left(u_{1}-u_{2}\right), \\
\frac{d v_{2}}{d t}=\tau\left(u_{2}-\gamma v_{2}\right),
\end{gathered}
$$


where $\alpha, \gamma, \tau$, and $K$ are parameters, $u_{1,2}(t)$ is the activator, and $v_{1,2}(t)$ is the inhibitor. The reason that we use the FHN neuron is that the equations are "standard" and "minimal" models for excitable systems in the following sense. The model contains no more than two variables, and does not describe a specific biochemical reaction: it can be used to describe both neural and cardiac dynamics.

\section{NUMERICAL ANALYSIS}

\section{A. Time evolution and firing diagram}

Employing the following parameters: $\tau=0.001, \gamma=0.0$, each element is excitable for $\alpha>0$, that is, a small but finite perturbation to the rest state $\left(u_{1}, v_{1}, u_{2}, v_{2}\right)=(0,0,0,0)$ leads to a large excursion (an excitation). Because of the excitable nature of the units, the rest state is a globally stable solution when the coupling is excitable, i.e., $K>0$. Indeed, stating from the rest state with a finite perturbation, these two elements excite and immediately synchronize. After synchronization, there are no input signals from the other element owing to diffusive coupling, and then, both elements immediately return to the rest states.

For an phase-repulsive coupling $K<0$, however, periodic excitations are observed in some parameter regions. A rich variety of cyclic firing patterns is found numerically, as depicted in Fig. 1. To characterize these periodic solutions, we introduce the following symbolic notation. In Fig. 1(a), after one element excites, the other element excites soon. After this successive excitations, both elements stay at the quiescence state for a while. These excitations and quiescence are repeated, and we symbolize this firing pattern as "AB-." Note that the code AB- and BA- correspond to the same periodic solution due to the invariance under the exchange of each element. In the same way, we characterize periodic solutions in Fig. 1 as "AB-AB-BA-BA-," "AB-BA-," and "ABA-BAB-."

Precisely speaking, these symbolic patterns can be obtained by the following criteria; when $\left(u_{i}(t)\right.$ $\left.=u_{c} \wedge d u_{i} / d t>0\right)$ is satisfied, we consider the excitation of element $i$ occurs, and generate symbol $\mathrm{A}$ for $i=1$ or $\mathrm{B}$ for $i=2$. If $\left[\left(d u_{1,2} / d t=0\right) \wedge\left(u_{2,1}<0\right)\right]$ or $\left[\left(d u_{2,1} / d t=0\right) \wedge\left(u_{1,2}<0\right)\right]$, we regard the system as quiescence state and generate the symbol "-," where $u_{c}=0.5$ is a threshold parameter. Even the simplest two-neuron model shows the enormous complexity that arises from interaction of nonlinear excitable elements. The firing pattern diagram in $(K, \alpha)$-parameter space is depicted in Fig. 2 from massive numerical computations by using above coding criteria.

To see dynamical behaviors, we calculate the interspike interval (ISI), which is defined as follows. Integrating the Eq. (1) numerically (we have used adaptive time step algorithm so called the fifth-order Runge-Kutta-Fehlberg formula because there exist slow and first variables in the system), we have a sequence of time $t_{i}$ at which excitation of element 1 occurs. The time sequence of the excitations can be obtained by $T=\left\{t_{i} \mid\left(u_{1}\left(t_{i}\right)=0\right) \wedge\left(d u_{1}\left(t_{i}\right) / d t>0\right)\right\}$. The ISI defined as the sequence of $\Delta t_{i}^{\prime}=t_{i+1}-t_{i}$ is often used as a characterization of neural activities [12]. After an initial transient disappears,
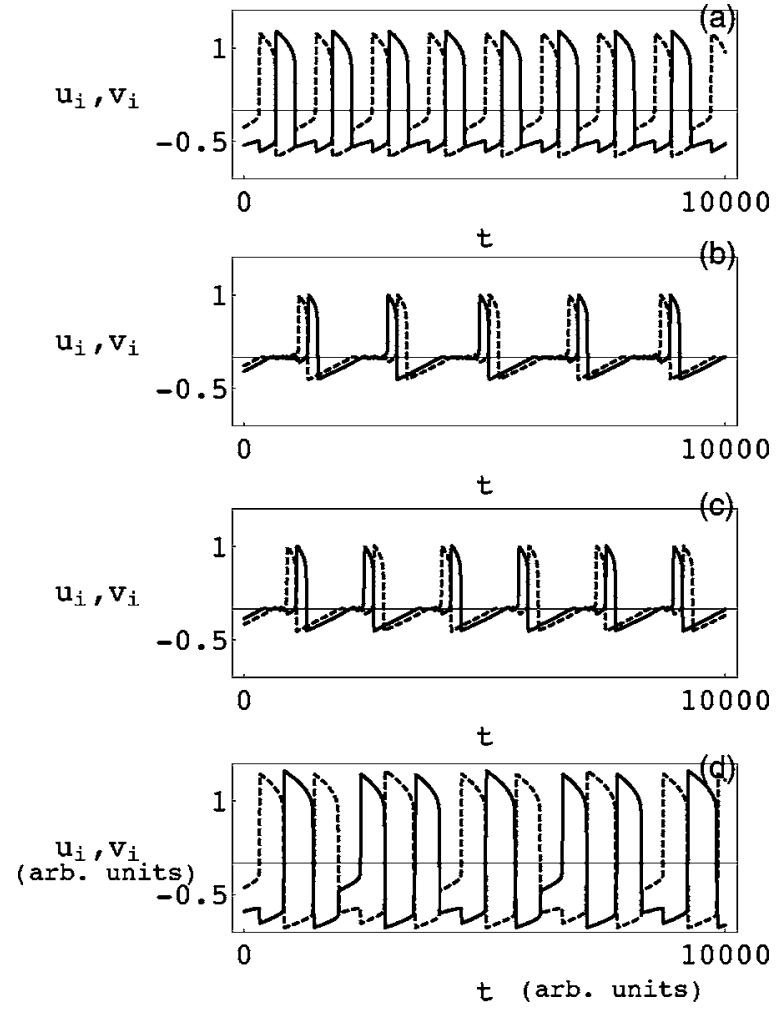

FIG. 1. Time evolution of a pair of FHN elements with phaserepulsive coupling. Periodic oscillations appear below a critical coupling strength $K \sim-0.01$. Typical periodic solutions are shown: (a) $K=-0.5$ : AB- firing pattern, (b) $K=-0.093$ : AB-AB-BA-BA-, (c) $K=-0.012$ : AB-BA-, (d) $K=-1.0$ : ABA-BAB-. The solid and broken lines represent $u_{1}(t)$ and $u_{2}(t)$, respectively. $\alpha=0.01$, $\tau=0.001, \gamma=0.0$

i.e., for $i \gg 1$, the superimposed ISI as a function of the parameter $K$ is plotted in Fig. 3. The ISI shows that the cyclic firing bifurcates to irregular one at $K=K_{\infty}$ by decreasing the coupling strength $K$. Further decreasing $K$, periodical firing appears again. The chaotic firing regions in $(K, \alpha)$-parameter space are shown in Fig. 2.

\section{B. Stability and bifurcation diagram}

Next, we investigate the origin of these firing patterns in the context of global bifurcation. In order to analyze the origin of the periodic solutions, we will first examine the stability of the rest state $\left(u_{1}, v_{1}, u_{2}, v_{2}\right)=(0,0,0,0)$. The Jacobian matrix of the rest state is given by

$$
\left(\begin{array}{cccc}
-\frac{K}{2}-\alpha & \tau & \frac{K}{2} & 0 \\
-1 & -\gamma \tau & 0 & 0 \\
\frac{K}{2} & 0 & -\frac{K}{2}-\alpha & \tau \\
0 & 0 & -1 & -\gamma \tau
\end{array}\right),
$$

and its eigenvalues are 

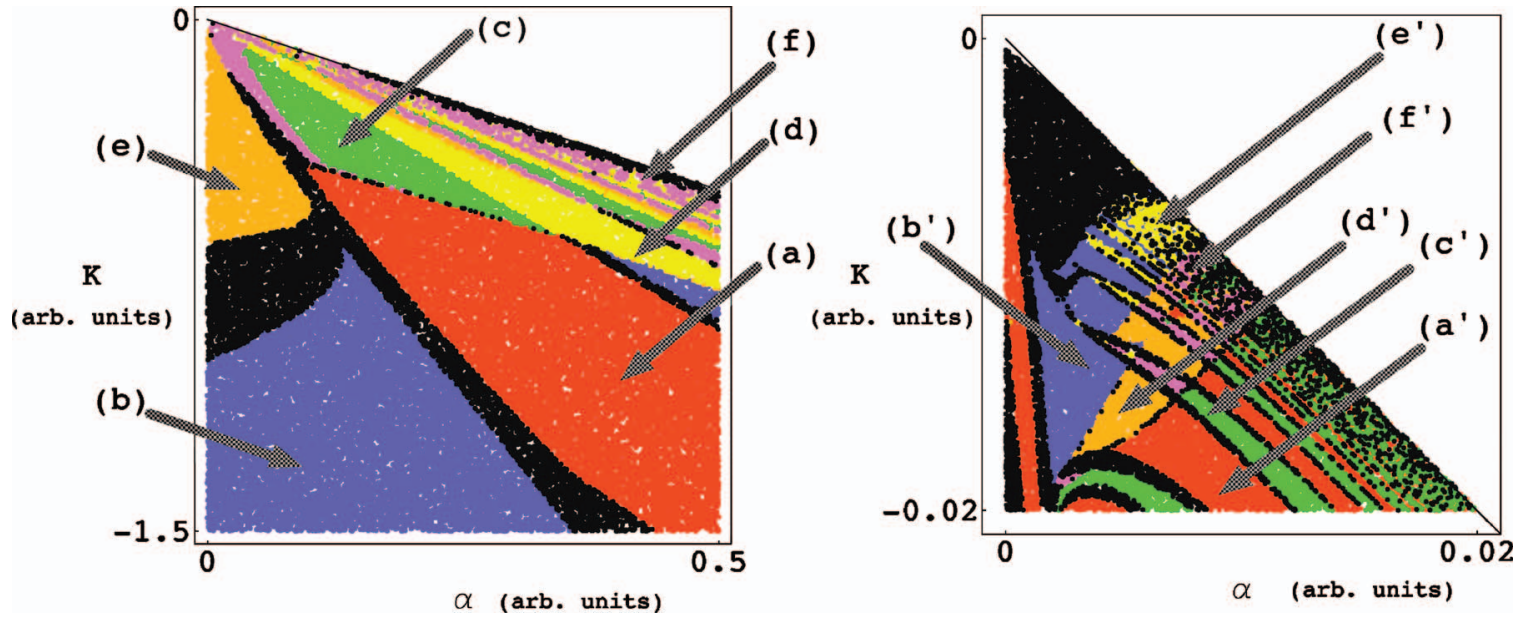

FIG. 2. (Color) Firing pattern diagram in $(K, \alpha)$-parameter space. The other parameter values are the same as in Fig. 1. The line represents the Hopf-bifurcation line: $K_{H P}=-\alpha$. Left: each colored dot corresponds to different firing pattern, (a) red: ABA-; (b) blue: ABA-BAB-; (c) green: AB-BA-; (d) orange: AB-; (e) yellow: AB-; (f) magenta: AB-BA-; and black: longer periodic or chaotic firing patterns. Right: the magnification of left figure gives clear evidence for the existence of a variety of attractors. $\left(\mathrm{a}^{\prime}\right)$ red: $\mathrm{AB}-\mathrm{BA}-;\left(\mathrm{b}^{\prime}\right)$ blue: $\mathrm{AB}(-)^{4} \mathrm{BA}(-)^{4} ;\left(\mathrm{c}^{\prime}\right)$ green: $\mathrm{AB}-;\left(\mathrm{d}^{\prime}\right)$ orange: $\mathrm{AB}(-)^{3} \mathrm{BA}(-)^{3} ;\left(\mathrm{e}^{\prime}\right)$ yellow: $\mathrm{AB}(-)^{4} ;\left(\mathrm{f}^{\prime}\right)$ magenta: $\mathrm{AB}(-)^{3}$; and black: longer periodic or chaotic firing patterns.

$$
\begin{gathered}
\lambda_{ \pm}^{1}=\left[-\alpha-\gamma \tau \pm \sqrt{-4 \tau+(\alpha-\gamma \tau)^{2}}\right] / 2, \\
\lambda_{ \pm}^{2}=\left[-K-\alpha-\gamma \tau \pm \sqrt{-4 \tau+(K+\alpha-\gamma \tau)^{2}}\right] / 2 .
\end{gathered}
$$

This means that the rest state becomes unstable at critical value $K_{H P}=-\alpha-\gamma \tau$ via the Hopf bifurcation [21].

To see the bifurcation more precisely, the solution branch that stems from the Hopf bifurcation (HP branch) is detected and continued numerically by using AUTO software [22], as shown in Fig. 4. We find that the Hopf bifurcation is subcritical. Furthermore, although the branch turns back at $K_{P D \cdot S N}=-0.003994$, the stability is not recovered. The numerical computation of Floquet exponents shows that the saddle-node and period-doubling bifurcations occur simultaneously at $K_{P D \cdot S N}$. The HP branch is always unstable for any coupling strength. Thus the periodic solutions obtained by numerical integration, as shown in Fig. 1, do not originate from the subscritical Hopf bifurcation, which stems from the rest state. And the HP branch does not directly connect to

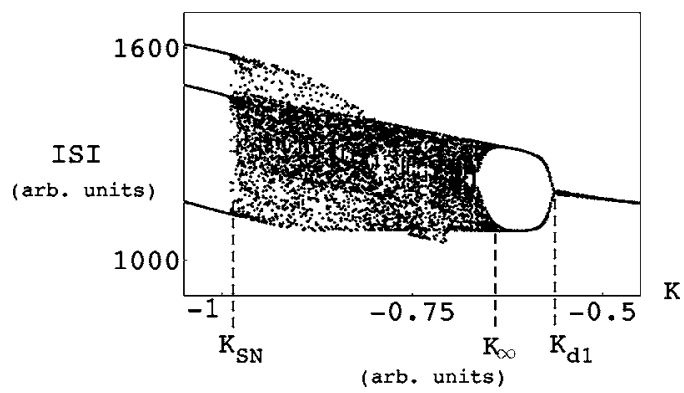

FIG. 3. Interspike interval as a function of coupling strength $K$. The chaotic firing appears after the accumulation of period doublings $K_{\infty} \sim-0.642$. Intermittent chaos occurs near $K_{S N}=-0.986$ through the saddle-node bifurcation. Both critical values are consistent with those obtained by AUTO. these periodic solutions. Indeed, the unstable periodic solution that stems from the Hopf bifurcation point shows the different firing pattern, antiphase excitation, which will be coded as A-B-, depicted in Fig. 4(b). The orbit starting from the A-B- solution at $K=-0.5$ with a tiny perturbation is attracted to the stable $\mathrm{AB}$ - pattern after a long transient.

We trace the branches of the solutions in Fig. 1 numeri-

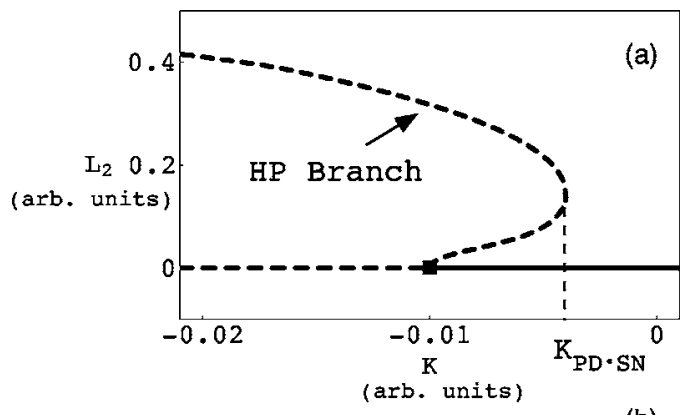

(b)

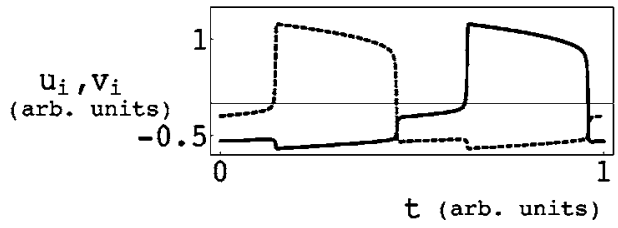

FIG. 4. (a) Bifurcation diagram of the rest state $\left(u_{1}, v_{1}, u_{2}, v_{2}\right)$ $=(0,0,0,0)$. The vertical axis shows the $L_{2}$ norm, $L_{2}$ $=1 / T \int_{0}^{T} \sqrt{\sum_{i=1,2} u_{i}^{2}+v_{i}^{2}} d t$, where $T$ is the period of solution. The rest state corresponding to $L_{2}=0$ becomes unstable after the Hopf bifurcation at $K=-0.01$. The solid line represents stable rest solution. The broken curve with $L_{2} \neq 0$ represents the solution branch emanating from the bifurcation. The branch is always unstable for any coupling strength $K$. (b) The unstable periodic solution on HP branch at $K=-0.5$. The time scale is normalized by its period $T=1068.3$. The lines and the other parameters are the same as in Fig. 1. 


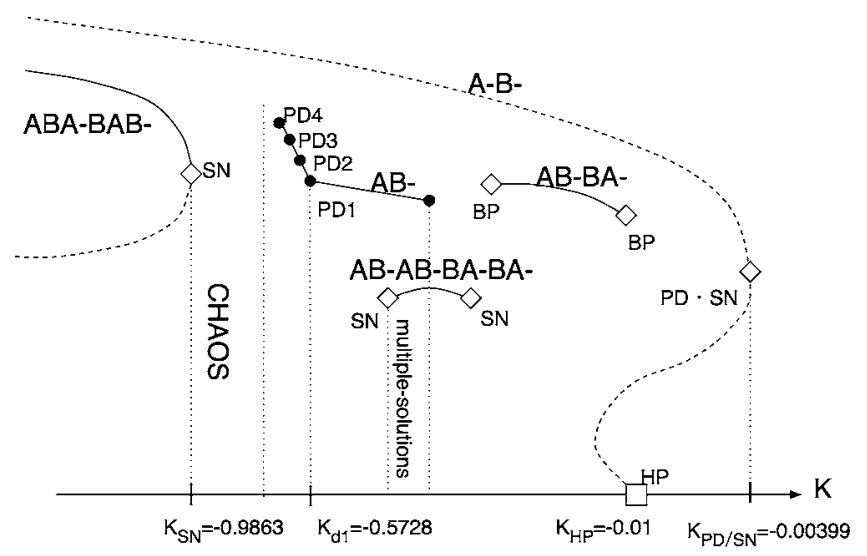

FIG. 5. Schematic bifurcation diagram obtained by AUTO. SN, HP, PD, and BP indicate a saddle-node, a Hopf, a period doubling, and a bifurcation point, respectively. Each stable branch corresponding to periodic solution is not connected to HP branch that emanates from the rest state via the subcritical Hopf bifurcation. The vertical axis shows the $L_{2}$ norm.

cally using AUTO. The schematic bifurcation diagram is depicted in Fig. 5. Decreasing $K$, the AB- solution becomes unstable at $K_{d 1} \sim-0.57288$ where the first period-doubling bifurcation occurs. Successive period-doubling bifurcations occur at $K_{d 2}=-0.630224, K_{d 3}=-0.639256$, $K_{d 4}=-0.641297$, and $K_{d 5}=-0.641735$. Below the accumulation point of the period doublings, $K_{\infty} \sim-0.642$, the chaotic firing pattern is clearly observed. The parameter values obtained by AUTO are consistent with those obtained through the superimposed ISI (see Fig. 3). The Feigenbaum constant estimated from $\left(K_{d 4}-K_{d 3}\right) /\left(K_{d 5}-K_{d 4}\right)$ is 4.65982 , and is consistent with theoretical prediction 4.6692 [23]. Although the interspike intervals are irregularly distributed below the accumulation point, the spiking pattern, i.e., the order of the excitations AB-, is preserved (Fig. 6). Further decreasing $K$, the ABA-BAB- solution appears via the saddle-node bifurcation at $K_{S N}=-0.9863$.

\section{Characterization of chaotic firing}

The Lyapunov characteristic exponents (LCEs) play a crucial role in the description of the behavior of dynamical systems. We calculate LCEs as a function of $K$ (see Fig. 7). The sum of the $p$ largest LCEs measures the average rates of divergence or convergence of $p$-dimensional phase-space volume. A positive maximum LCE is obtained in the chaotic region $K_{S N}=-0.9863<K<K_{\infty}=-0.642$, that is also consistent with both the superimposed ISI (Fig. 3) and the global bifurcation diagram obtained by AUTO (Fig. 5). In addition, a single positive LCE exists in the chaotic region, meaning that hyperchaos does not observe, and Lyapunov dimension $D$ of the attractor is about $D \sim 2.0003$ throughout the chaotic region.

For coupling strength slightly larger than $K_{S N}$, an intermittent behavior is observed, where the time evolution is a mixture of AB- and ABA-BAB- firing patterns. Since the ABA$\mathrm{BAB}$ - periodic solution disappears through the saddle-node bifurcation at $K_{S N}, \mathrm{ABA}-\mathrm{BAB}$ - and $\mathrm{AB}$ - firing patterns cor-
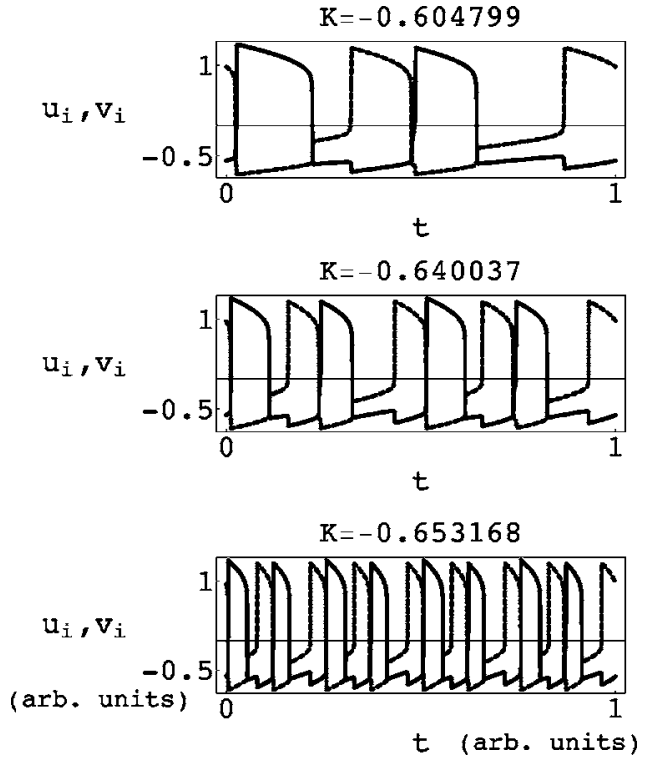

FIG. 6. The solutions at period-doubling bifurcations obtained by AUTO. The time is normalized by its period. The period of each solution is $T=2407.42,4827.59$, and 9660.59 from top to bottom, respectively.

respond to laminar and turbulent states, respectively [23]. We plot the average time between bursts (AB- firing) as a function of $K-K_{H P}$, and obtain the corresponding exponent as $-1 / 2$ (Fig. 8). Thus the intermittency is type I, and the result is consistent with the bifurcation analysis.

\section{Multistability and the existence of firings with long interval}

Careful numerics shows that multiple stable periodic solutions exist in the parameter region -0.09400 $<K<-0.09322$. Each solution is coded as AB-AB-BA-BAand $\mathrm{AB}-$, respectively (see Fig. 2). This asymptotic behavior in the parameter region depends on an initial condition.

For smaller values of $K$, we find complicated firing patterns, which consist of successive excitations of elements and long intervals. $\mathrm{AB}(-)^{n}$ and $\mathrm{AB}(-)^{n} \mathrm{BA}(-)^{n}$ firing patterns as shown in Fig. 9, for example, emanate via saddle-node bifurcations, where code $(-)^{n}$ represents the long interval in which a tiny antiphase oscillation is observed. The tiny oscillation will be corresponding to the subthreshold oscillation [11] in neural activity. There are many stable periodic solu-

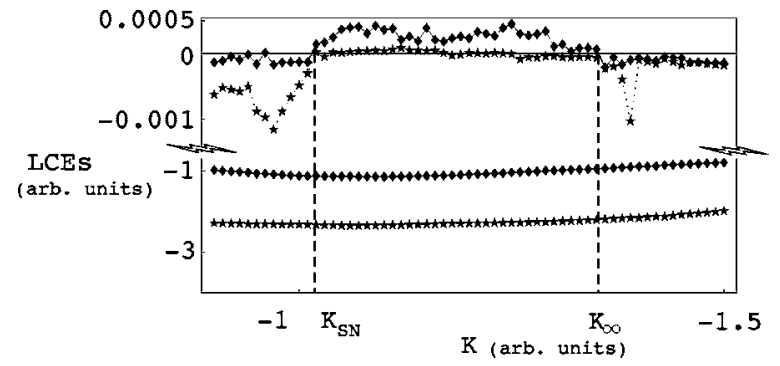

FIG. 7. Lyapunov characteristic exponents as a function of $K$ is plotted. One positive Lyapunov exponent exists in the chaotic region, meaning that there is no hyperchaotic attracter. 


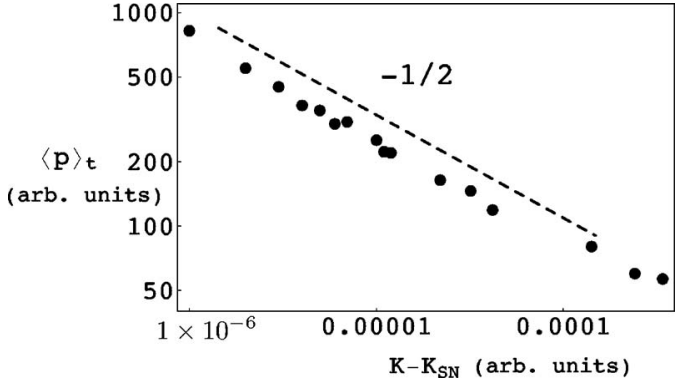

FIG. 8. The log-log plot of the time averaged life time of ABABAB- laminar oscillation as a function of the distance from the critical point $K-K_{H P}$. The $-1 / 2$ exponent indicates type I intermittency, which is consistent with the result of numerical bifurcation analysis.

tions that have a long period near the Hopf-bifurcation line $K_{H P}=-\alpha-\gamma \tau$. However, these solutions are not directly connected to the Hopf bifurcation. The solutions suddenly emanate near the bifurcation line. Moreover, each solution branch is isolated from another, and is not connected to each other. Although we cannot check all of the origins of these periodic solutions, most of the solutions emanate suddenly via saddle-node bifurcations.

\section{SUMMARY}

We have found many firing patterns, for example, AB-, AB-BA-, AB-AB-BA-BA-, and ABA-ABA-, in a pair of FHN elements with phase-repulsive coupling. Through numerical continuation of these solutions, we have shown that these firing patterns correspond to periodic solutions mostly emerged from the saddle-node bifurcations. The stability analysis of the rest state shows that the Hopf bifurcation occurs at $K_{H P}=-\alpha-\gamma \tau$. However, this Hopf bifurcation is subcritical, and the solution branch that stems from the bifurcation is unstable. Furthermore, although the HP branch turns back at $K=K_{P D \cdot S N}$ via the saddle-node bifurcation, the stability is not recovered because of the simultaneous occurrence of the period-doubling bifurcation.

It is also shown that a pair of excitable FHN neurons exhibits the chaotic firing that appears after the accumulation of the period-doubling bifurcations from the $\mathrm{AB}$ - solution. This chaotic firing is terminated by the sudden emergence of the periodic ABA-BAB- solution. Since the ABA-BAB- solution appears through the saddle-node bifurcation, the chaotic firing shows a type I intermittent behavior near the critical point. In short, the simple excitable FHN elements can potentially produce a wide variety of temporal patterns

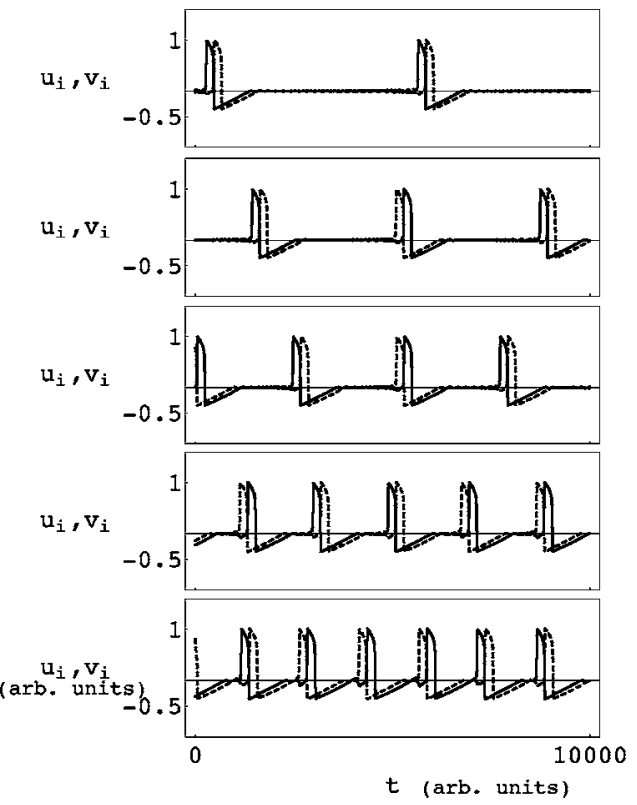

FIG. 9. Emergence of periodic solution with a long interval via saddle-node bifurcation. The period of the solution becomes very long as $K$ approaches the bifurcation line $-\alpha-\gamma \tau$. $K=-0.0105$, $-0.011,-0.012,-0.015$, and -0.025 from top to bottom, respectively.

through mutual inhibition. Although the model we have studied is much simpler than those of previous works [5-8], various dynamical behaviors have emerged. These facts shed some light on a neural activity in the brain where periodic behaviors are often observed.

In a pair of the FHN neurons, there are parameter regions in which multistable periodic solutions exist. Preliminary simulations of a large number of FHN excitable elements show the coexistence of many stable periodic solutions with fixed parameters. Starting from a state that is close to one of these limit cycles, the system will go into the respective limit cycle. Therefore the excitable elements with phase-repulsive coupling may be used to store memories in limit cycles.

\section{ACKNOWLEDGMENTS}

We would like to thank David Terman for his useful comments. We also would like to thank Makoto Iima for interesting discussions. One of the authors (T.Y.) acknowledges partial support from Grant-in-Aids for Scientific Research from the Ministry of Education, Science, and Culture of Japan.
[1] A. N. Zaikin and A. M. Zhabotinsky, Nature (London) 255, 535 (1970)

[2] A. T. Winfree, Science 175, 634 (1972).

[3] S. Kondo and R. Asai, Nature (London) 376, 765 (1995).

[4] K. G. Pearson, Curr. Opin. Neurobiol. 10, 649 (2000).
[5] H. Fujii and I. Tsuda, Lect. Notes Comput. Sci. 3146, 140 (2004).

[6] V. E. Bondarenko and T. R. Chay, Phys. Rev. E 58, 8036 (1998).

[7] K. Tateno, H. Tomonari, H. Hayashi, and S. Ishizuka, Int. J. 
Bifurcation Chaos Appl. Sci. Eng. 14, 1559 (2004).

[8] R. Dogaru and L. O. Chua, Int. J. Bifurcation Chaos Appl. Sci. Eng. 8, 211 (1998).

[9] G. Matsumoto, K. Aihara, Y. Hanyu, N. Takahashi, S. Yoshizawa, and J. Nagumo, Phys. Lett. A 123, 162 (1987).

[10] L. Glass and R. Perez, Phys. Rev. Lett. 48, 1772 (1982).

[11] D. T. Kaplan, J. R. Clay, T. Manning, L. Glass, M. R. Guevara, and A. Shrier, Phys. Rev. Lett. 76, 4074 (1996).

[12] J. R. Clay, J. Comput. Neurosci. 15, 43 (2003).

[13] T. Yanagita, Y. Nishiura, and R. Kobayashi, Phys. Rev. E 71, 036226 (2005).

[14] Y. Kuramoto, Chemical Oscillations, Waves, and Turbulence (Springer, New York, 1984).

[15] G. Balázsi, A. Cornell-Bell, A. B. Neiman, and F. Moss, Phys. Rev. E 64, 041912 (2001).

[16] Y. D. Sato and M. Shiino, Phys. Rev. E 66, 041903 (2002).
[17] D. Terman, N. Kopell, and A. Bose, Physica D 117, 241 (1998).

[18] S. Kunec and A. Bose, Phys. Rev. E 63, 021908 (2001).

[19] R. FitzHugh, Biophys. J. 1, 445 (1961).

[20] J. Nagumo, S. Arimoto, and S. Yoshizawa, Proc. IRE 50, 2061 (1962).

[21] C. Rocşoreanu, A. Georgescu, and N. Giurgițeanu, The FithzHugh-Nagumo Model: Bifurcation and Dynamics, Mathematical Modeling: Theory and Applications (Kluwer Academic Publishers, Dordrecht, 2000).

[22] E. J. Doedel, R. C. Paffenroth, A. R. Champneys, T. F. Fairgrieve, Y. A. Kuznetsov, B. Sandstede, and X. Wang, Technical Report, Caltech, 2001.

[23] E. Otto, Chaos in Dynamical Systems (Cambridge University Press, New York, 1993). 